\title{
Acute Phase Proteins Are Baseline Predictors of Tuberculosis Treatment Failure
}

\author{
Nathella Pavan Kumar ${ }^{1 *}$, Kadar Moideen ${ }^{2}$, Arul Nancy ${ }^{2}$, Vijay Viswanathan ${ }^{3}$, \\ Kannan Thiruvengadam ${ }^{1}$, Shanmugam Sivakumar ${ }^{1}$, Syed Hissar ${ }^{1}$, \\ Hardy Kornfeld ${ }^{4}$ and Subash Babu ${ }^{1,5}$
}

${ }^{1}$ Indian Council of Medical Research (ICMR)-National Institute for Research in Tuberculosis, Chennai, India, ${ }^{2}$ National Institute for Research in Tuberculosis, International Center for Excellence in Research, National Institutes of Health, Chennai, India, ${ }^{3}$ Department of Diabetology, Prof. M. Viswanathan Diabetes Research Center, Chennai, India, ${ }^{4}$ Department of Medicine, University of Massachusetts Medical School, Worcester, MA, United States, ${ }^{5}$ Laboratory of Parasitic Diseases, National Institute of Allergy and Infectious Diseases, National Institutes of Health, Bethesda, MD, United States

OPEN ACCESS

Edited by:

Christoph Hölscher, Research Center Borstel (LG), Germany

Reviewed by: Marco Pio La Manna, University of Palermo, Italy

Rannakoe Lehloenya, University of Cape Town, South Africa

*Correspondence: Nathella Pavan Kumar pavankumarn@nirt.res.in

Specialty section:

This article was submitted to Microbial Immunology,

a section of the journal

Frontiers in Immunology

Received: 29 June 2021 Accepted: 25 October 2021 Published: 15 November 2021

Citation:

Kumar NP, Moideen K, Nancy A, Viswanathan $V$, Thiruvengadam $K$, Sivakumar S, Hissar S, Kornfeld $H$ and Babu S (2021) Acute Phase Proteins Are Baseline Predictors of

Tuberculosis Treatment Failure.

Front. Immunol. 12:731878 doi: 10.3389/fimmu.2021.731878
Systemic inflammation is a characteristic feature of pulmonary tuberculosis (PTB). Whether systemic inflammation is associated with treatment failure in PTB is not known. Participants, who were newly diagnosed, sputum smear and culture positive individuals with drug-sensitive PTB, were treated with standard anti-tuberculosis treatment and classified as having treatment failure or microbiological cure. The plasma levels of acute phase proteins were assessed at baseline (pre-treatment). Baseline levels of C-reactive protein (CRP), alpha-2 macroglobulin (a2M), Haptoglobin and serum amyloid $\mathrm{P}$ (SAP) were significantly higher in treatment failure compared to cured individuals. ROC curve analysis demonstrated the utility of these individual markers in discriminating treatment failure from cure. Finally, combined ROC analysis revealed high sensitivity and specificity of 3 marker signatures comprising of CRP, a2M and SAP in distinguishing treatment failure from cured individuals with a sensitivity of $100 \%$, specificity of $100 \%$ and area under the curve of 1 . Therefore, acute phase proteins are very accurate baseline predictors of PTB treatment failure. If validated in larger cohorts, these markers hold promise for a rapid prognostic testing for adverse treatment outcomes in PTB.

Keywords: tuberculosis, acute phase proteins, biomarker, inflammation, TB treatment

\section{INTRODUCTION}

Systemic inflammation is a characteristic hallmark of pulmonary tuberculosis (PTB) (1-3). Systemic inflammation is typically characterized by elevations in the levels of acute phase proteins, including C-reactive protein (CRP), apha-2 macroglobulin (a2M), haptoglobin (Hp) and serum amyloid P (SAP) (4-7). Several studies have associated increased circulating levels of these acute phase proteins with PTB and indeed, CRP is often used a point-of-care test to aid in the diagnosis of PTB (8-13). Studies have also reported that haptoglobin is clinically a relevant host biomarker for TB diagnosis and disease progression (14). 
While standard TB treatment is associated with high rates of favorable outcomes (recurrence free microbiological cure), a sizeable proportion of patients experience adverse outcomes in the form of treatment failure or recurrence after treatment (15). We have previously reported that plasma chemokine signatures (16), as well as Matrix metalloproteinases (MMPs) and tissue inhibitors of matrix metalloproteinases (TIMPs) (17) can be used as novel biomarkers for predicting adverse treatment outcomes like failure, relapse, and death in individuals with PTB. The host factors that drive this dichotomy in drug - sensitive TB are ill defined (18). We hypothesized that TB treatment failures would be driven by heightened systemic inflammation at baseline. To test this hypothesis, we examined the baseline levels of acute phase proteins in a nested case-control study of TB treatment failure versus cure in a cohort of PTB individuals in Chennai, India. Our results show that acute phase proteins are baseline predictors of treatment failure.

\section{MATERIALS AND METHODS}

\section{Ethics Statement}

This study was approved by the Ethics Committees of the Prof. M. Viswanathan Diabetes Research Center (ECR/51/INST/TN/ 2013/MVDRC/01) and NIRT (NIRT-INo:2014004). Informed written consent was obtained from all participants. All the methods were performed in accordance with the relevant institutional ethical committee guidelines.

\section{Study Population}

Participants were enrolled from the Effect of Diabetes on Tuberculosis Severity (EDOTS) study, a prospective cohort study conducted in Chennai, India (19). The inclusion criteria were new smear and culture positive adults between 20 and 75 years of age. The exclusion criteria were previous TB history or treatment, drug resistant $\mathrm{TB}$, more than one week of $\mathrm{TB}$ treatment currently, pregnancy or lactation, HIV positivity or on immunosuppression. The diagnosis of pulmonary TB was established by positive sputum culture on solid media with compatible chest $\mathrm{x}$-ray. Anti-TB treatment (ATT) was managed by government clinics in Chennai according National Tuberculosis Elimination Program standards, which is based on the Directly Observed Treatment Short Course (DOTS) therapy. Participants were followed up monthly through the six-month course of treatment. We conducted a nested case-control study with microbiological TB treatment failure matched in a 1:2 ratio to microbiological cure. Cure was defined as negative sputum cultures at months 5 and 6 of treatment. Treatment failure was defined as positive sputum culture at months 5 or 6 . There was a total of 18 treatment failures and 36 cured controls. Case control matching was carried out on the basis of age, gender, body mass index and diabetic status. Peripheral blood was collected in heparinized tubes. Following centrifugation, plasma was collected and stored at $-80^{\circ} \mathrm{C}$ till further analysis. Sample collection was performed at baseline (before treatment initiation) in all participants.

\section{Acute Phase Proteins}

Plasma levels of alpha-2 macroglobulin (A2M), C-reactive protein (CRP), haptoglobin and Serum Amyloid P (SAP) were measured using a Milliplex MAP Human CVD Panel Acute Phase magnetic bead panel 3 from Millipore, using a multiplex platform according to the manufacturer's instructions. The lowest detection limits for acute phase proteins was as follows: alpha-2 macroglobulin (A2M); $0.49 \mathrm{ng} / \mathrm{mL}$; C-reactive protein (CRP), $0.05 \mathrm{ng} / \mathrm{mL}$; haptoglobin, $0.06 \mathrm{ng} / \mathrm{mL}$; and Serum Amyloid P(SAP) $0.06 \mathrm{ng} / \mathrm{mL}$.

\section{Statistical Analysis}

Geometric means (GM) were used for measurements of central tendency. Differences between the two groups were analyzed using the Mann-Whitney test. Receiver Operator Characteristics (ROC) curves was designed to test the power of each candidate biomarker to distinguish treatment failures from cured individuals. Analyses were performed using Graph-Pad PRISM Version 9.0. P values < 0.05 were considered statistically significant. Computation and selection of optimal biomarker combinations by integrative ROC were analysed using freely available web application (http:// CombiROC.eu) CombiROC v.1.2. Classification and regression trees (CART) model were employed to identify the cut-off for the biomarkers which separate the TB treatment failure and cure. The analysis was done using the $\mathrm{R}$ ( $\mathrm{R}$ Foundation for Statistical Computing, Vienna, Austria) software.

\section{RESULTS}

\section{Study Population}

The demographics of the study population are shown in Table 1. The median age was 45 (interquartile range [IQR] 38-51) years for treatment failures and 45 (IQR 39-53) years for cured individuals. There were no significant differences in gender, BMI, diabetic status, lipid profile, smoking or alcohol use (Table 1). There were also no differences in smear or culture grades or presence of cavities between the two groups (Table 1).

\section{Treatment Failures in PTB Are Characterized by Increased Systemic Inflammation}

To assess baseline systemic inflammation in treatment failure and cured individuals, we measured the levels of acute phase proteins at baseline (pre-treatment). As shown in Figure 1, the levels of CRP (Geometric Mean (GM) of $18.34 \mathrm{ng} / \mathrm{ml}$ in failure versus $2 \mathrm{ng} / \mathrm{ml}$ in cure), A2M (GM of $1866 \mathrm{ng} / \mathrm{ml}$ in failure versus $23.4 \mathrm{ng} / \mathrm{ml}$ in cure), $\mathrm{Hp}$ ( $\mathrm{GM}$ of $91.2 \mathrm{ng} / \mathrm{ml}$ in failure versus $72.9 \mathrm{ng} / \mathrm{ml}$ in cure) and SAP ( $\mathrm{GM}$ of $1.02 \mathrm{ng} / \mathrm{ml}$ in failure versus $0.22 \mathrm{ng} / \mathrm{ml}$ in cure) were significantly higher in cases compared to controls. Thus, adverse treatment outcomes in PTB are associated with increased baseline levels of acute phase proteins.

\section{Treatment Failures in PTB Are Marked by a Three-Marker Signature of Acute Phase Proteins}

To determine if we could utilize acute phase proteins as individual biomarkers for treatment failures versus cured 
TABLE 1 | Demographic and clinical characteristics of the study population.

\begin{tabular}{|c|c|c|c|}
\hline & Cure $(n=36)$ & Treatment failures $(n=18)$ & Sig. \\
\hline Age (in Years) & $45.0(38.0-50.5)$ & $45.0(39.0-53.0)$ & 0.818 \\
\hline \multicolumn{4}{|l|}{ Gender } \\
\hline Female & 7 (19.4) & $2(11.1)$ & \multirow[t]{2}{*}{0.439} \\
\hline Male & 29 (80.6) & $16(88.9)$ & \\
\hline BMl & $17.0(15.4-19.4)$ & $16.8(14.9-19.6)$ & 0.646 \\
\hline \multicolumn{4}{|l|}{ Diabetes } \\
\hline Non-Diabetes & $14(38.9)$ & $5(27.8)$ & \multirow[t]{2}{*}{0.420} \\
\hline Diabetes & $22(61.1)$ & $13(72.2)$ & \\
\hline Cough Duration & $5.0(3.0-8.0)$ & $4.5(4.0-8.0)$ & 0.661 \\
\hline \multicolumn{4}{|l|}{ Cough } \\
\hline Absence & $2(5.6)$ & $0(0)$ & \multirow[t]{2}{*}{0.308} \\
\hline Presence & $34(94.4)$ & $18(100)$ & \\
\hline \multicolumn{4}{|l|}{ Dyslipidaemia } \\
\hline Absence & $36(100)$ & $18(100)$ & \multirow[t]{2}{*}{ NA } \\
\hline Presence & $0(0)$ & $0(0)$ & \\
\hline \multicolumn{4}{|l|}{ Smoking } \\
\hline Never & $20(55.6)$ & $6(33.3)$ & \multirow[t]{2}{*}{0.123} \\
\hline Past/Current & $16(44.4)$ & $12(66.7)$ & \\
\hline \multicolumn{4}{|l|}{ Alcohol } \\
\hline Never & $13(36.1)$ & $4(22.2)$ & \multirow[t]{2}{*}{0.300} \\
\hline Past/Current & $23(63.9)$ & $14(77.8)$ & \\
\hline \multicolumn{4}{|l|}{ Cavity } \\
\hline Absence & $22(61.1)$ & $10(55.6)$ & \multirow[t]{2}{*}{0.345} \\
\hline Presence & $14(38.9)$ & $8(44.4)$ & \\
\hline \multicolumn{4}{|l|}{ Smear } \\
\hline $1+$ & $25(69.4)$ & $10(55.6)$ & \multirow[t]{3}{*}{0.561} \\
\hline $2+$ & $9(25)$ & 6 (33.3) & \\
\hline $3+$ & $2(5.6)$ & $2(11.1)$ & \\
\hline \multicolumn{4}{|l|}{ Culture } \\
\hline $1+$ & $11(30.6)$ & 6 (33.3) & \multirow[t]{3}{*}{0.185} \\
\hline $2+$ & 12 (33.3) & $2(11.1)$ & \\
\hline $3+$ & $13(36.1)$ & $10(55.6)$ & \\
\hline
\end{tabular}

Values were presented as n (\%) and median (first - third quartile); Fisher Exact and MannWhitney test were used to check the significance

individuals, we performed individuals ROC analysis on acute phase proteins. As shown in Figure 2A, ROC analysis of CRP (Sensitivity 94\%, Specificity $86 \%$ and AUC=0.9213), Hp (Sensitivity 66\%, Specificity $71 \%$ and $\mathrm{AUC}=0.7461$ ) and SAP (Sensitivity 94\%, Specificity $88 \%$ and AUC=0.9715) showed significantly high AUC with sensitivity and specificity, especially for A2M, which exhibited $100 \%$ sensitivity and specificity. To determine if we could derive a signature of acute phase proteins that could be used as a biomarker for treatment failures versus cured individuals, we performed combined ROC analysis on 3 acute phase proteins. As shown in Figure 2B, combiROC analysis of CRP, A2M and SAP exhibited a high AUC (1) with $100 \%$ sensitivity and specificity in differentiating treatment failures from microbiologic cures. Thus, treatment failures are marked by a three-marker signature of acute phase proteins.

\section{Biomarkers Discriminating Favorable From Unfavorable TB}

Classification and regression trees (CART) models were employed to identify the cut-off for the biomarkers which separate the treatment failures from cure. As input for tree construction, we used data on all the markers and selected the most relevant biomarker that classifies the group more accurately
(Figure 3). Briefly, the data set formed a parent node, which contains the whole population. The best peak to separate the data set was selected. As shown in Figure 3, CRP with a cut-off value of $7.8 \mathrm{ng} / \mathrm{ml}$ with AUC:0.91, A2M with a cut-off value of $90 \mathrm{ng} /$ $\mathrm{ml}$ with AUC:1, Hp with a cut-off value of $92 \mathrm{ng} / \mathrm{ml}$ with AUC:0.74 and SAP with a cut-off value of $0.63 \mathrm{ng} / \mathrm{ml}$ with AUC:0.89 was able distinguish failure vs cure. This CART analysis was able to demonstrate that APPs such as CRP and a2M act as a sensitive diagnostic immune biomarker for prediction of TB treatment outcomes.

\section{DISCUSSION}

Tuberculosis patients are highly heterogeneous when it comes to the extent of disease, immune activation and vulnerability to treatment failure $(18,20)$. Therefore, tools guiding individual management and treatment are likely to increase cure rates and be more cost-effective, which would also largely facilitate the clinical trial evaluation of new drugs and regimens. Treatment is prolonged, and patients on ATT often experience treatment failure for various reasons (15). Therefore, there is an urgent need to identify host mechanisms that drive treatment failure, recurrence and death, since these factors in addition to bacterial factors, contribute majorly to adverse treatment outcomes (21). Greater understanding of the host processes underlying this phenomenon could provide clues to developing biomarkers of adverse treatment outcomes in TB.

Our study examined the occurrence of systemic inflammation in a well characterized cohort of PTB individuals followed up until completion of treatment and classified as treatment failures or microbiological cures. Our data reveal that systemic inflammation, as measured by the plasma levels of acute phase protein, at baseline are significant predictive biomarkers of treatment failure. Data from various studies have collectively reported that CRP exhibited high sensitivity (93\%) and moderate specificity $(60 \%)$ in distinguishing active $\mathrm{TB}$ from other conditions (22). Indeed, CRP has been recommended as a simple, point-of-care to aid in the diagnosis of PTB (8-13). Therefore, our data on CPR being significantly associated with treatment failures is not surprising. A2M, Hp and SAP have also been reported to be useful biomarkers for TB diagnosis $(14,23-$ 26). Our data clearly demonstrate that all the 4 acute phase proteins are present at higher levels in failures compared to cured individuals. Our ROC analysis data confirms the utility of CRP, A2M and SAP as potentially valuable biomarkers for treatment failure. Finally, we derive a three-biomarker signature comprising of CRP, A2M and SAP that provides $100 \%$ sensitivity and $100 \%$ specificity in distinguishing treatment failures from cured individuals in our cohort. While systemic inflammation in TB is not a new phenomenon, this to our knowledge is one of the first reports on systemic inflammation being an underlying player in adverse treatment outcomes in PTB.

Our study groups were matched for age, gender, body mass index and diabetic status. Other confounders such as smoking, alcohol use, lipid status, smear and culture grade did not exhibit 


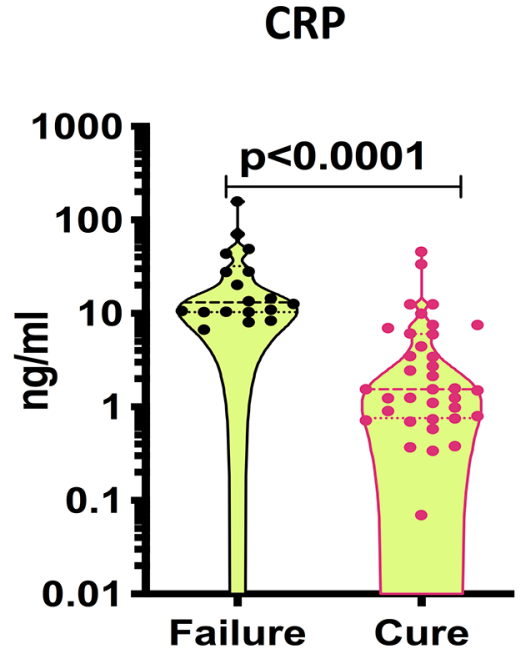

Haptoglobin

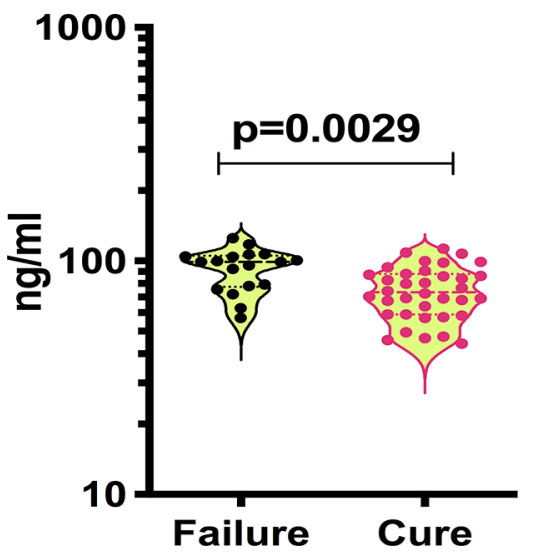

a-2-Macroglobulin

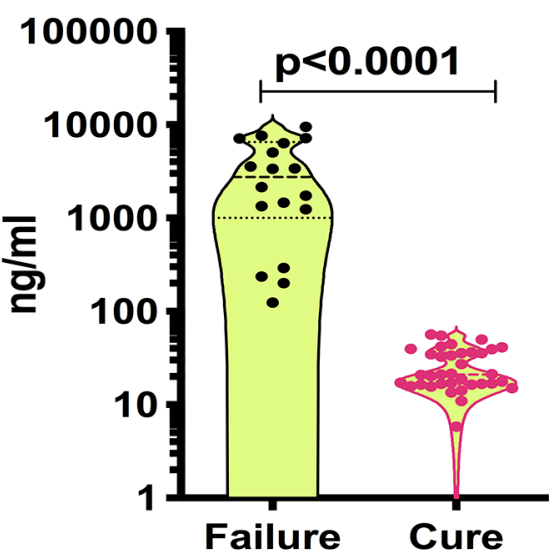

Serum Amyloid $\mathbf{P}$

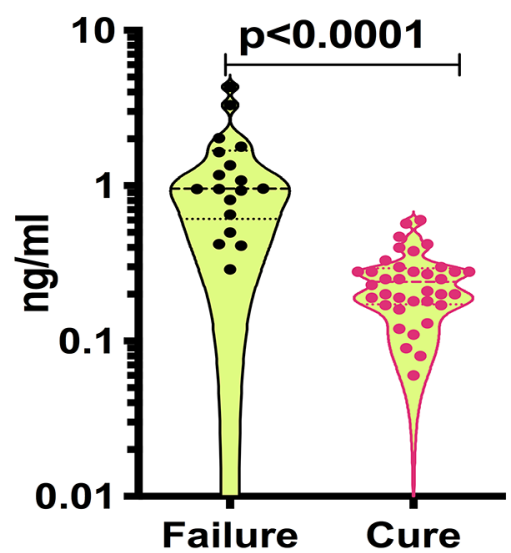

FIGURE 1 | Elevated baseline plasma levels of APPs in TB treatment failure. The baseline plasma levels of APPs were measured in failure ( $n=18$ ) and cure ( $n=36$ ). The data are represented as violin plots with each circle representing a single individual. P values were calculated using the Mann-Whitney test with Holm's correction for multiple comparisons.

any significant differences. In addition, our data provide cut off values in $\mathrm{pg} / \mathrm{ml}$ for the biomarkers of CRP, A2M and SAP to be used for classification of individuals at risk of treatment failure. We anticipate validating these cut off values in future cohorts. Therefore, our study provides an important advance in the field TB biomarkers by providing a potential signature of high utility in baseline stratification of high risk individuals for TB treatment. Our study adds to the growing list of biomarkers that can be used to predict adverse treatment outcomes at pretreatment timepoints. Further confirmation of these markers in diverse geographic and ethnic setting would add value to their utility in this field. In addition, studies also reported that in other respiratory chronic diseases like chronic obstructive pulmonary disease, asthma and pulmonary fibrosis also persistent inflammation is seen within the respiratory tract underlies the pathogenesis of numerous chronic pulmonary diseases $(27,28)$

Our study has certain limitations. The sample size was moderate, the study was performed in a single cohort without external validation and cause-effect relationships were not determined. Our study also has not investigated the causes of the inflammatory condition which is due to the tuberculosis, or which may be due to comorbid conditions. Nevertheless, this data is of major importance in designing future studies to elucidate host biomarkers of adverse treatment outcomes, which would be enormously beneficial in eliminations strategies for PTB. Moreover, our study also contributes to the growing understanding of the pathogenesis of host responses that govern treatment outcomes in TB. 
A

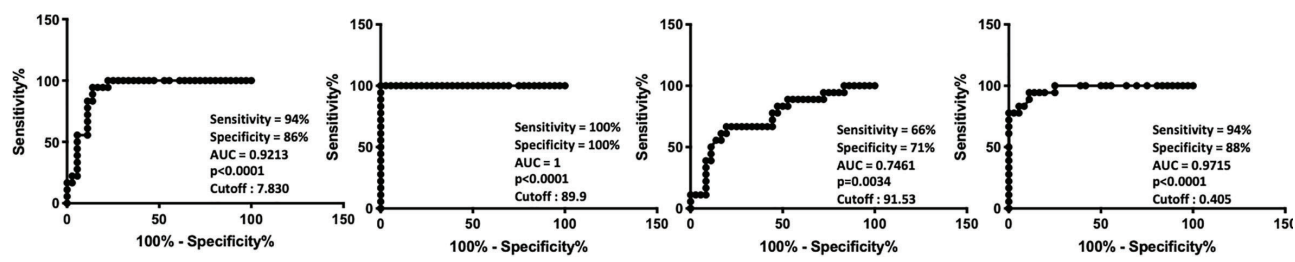

B

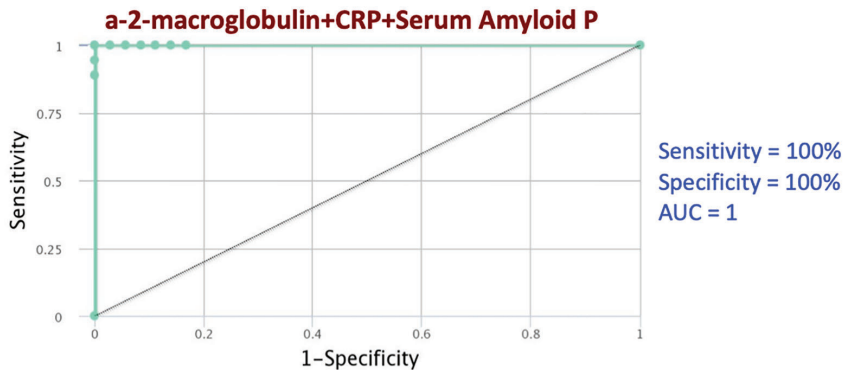

FIGURE 2 | ROC analysis to estimate the discriminatory power of APPs in TB treatment failure. (A) ROC analysis to estimate the sensitivity, specificity and AUC was performed using a-2M, CRP, Hp and SAP to estimate the capacity of these markers to distinguish individuals with failure vs. Cure. (B) Combination of ROC model analysis shows the APPs that exhibited the highest accuracy in discriminating failure and cure.
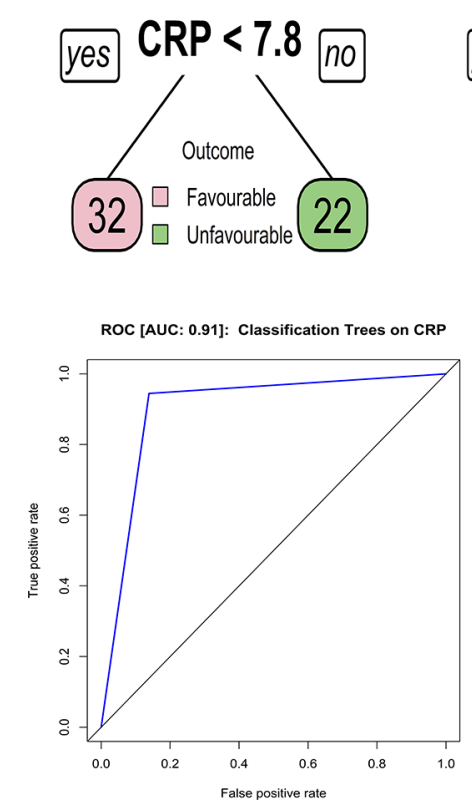

yes a2macroglobulin $<90$

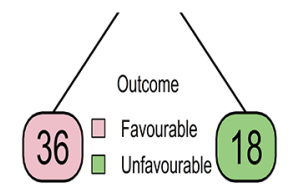

no yes Haptoglobin < 92

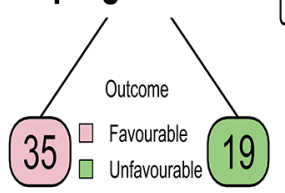

\section{SerumAmyloidP $<0.63$ no}
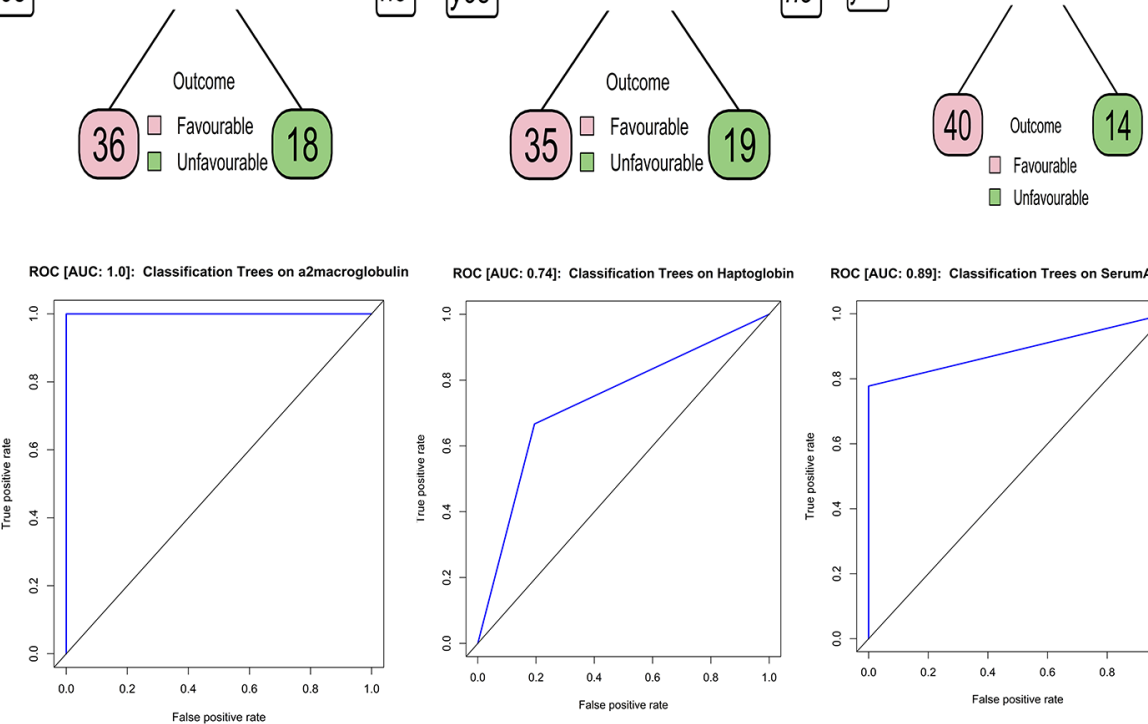

ROC [AUC: 0.74]: Classification Trees on Haptoglobin
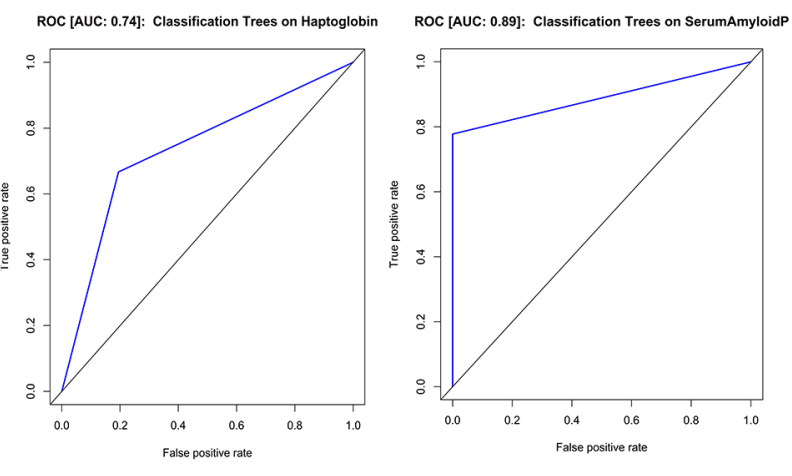

FIGURE 3 | Identification of biomarkers showing the strongest associations with active TB disease. CART model analysis shows the APPs that exhibited the highest accuracy in discriminating TB treatment failure from cure. Receiver operator characteristics curves were employed to quantify the accuracy of single biomarkers. 


\section{DATA AVAILABILITY STATEMENT}

The original contributions presented in the study are included in the article/supplementary material. Further inquiries can be directed to the corresponding author.

\section{ETHICS STATEMENT}

The studies involving human participants were reviewed and approved by the NIRT-Ethics committee. The patients/ participants provided their written informed consent to participate in this study.

\section{AUTHOR CONTRIBUTIONS}

Designed the study (SB, NK). Conducted experiments (NK, KM, $\mathrm{AN})$. Acquired data (NK, KT). Analyzed data (NK, KT). Contributed reagents and also revised subsequent drafts of the manuscript (SH, SS, HK, SB). Responsible for the enrolment of participant and also contributed to acquisition and interpretation of clinical data (VV, $\mathrm{SH}, \mathrm{HK})$ Wrote the manuscript (SB, NK). All authors read and approved the final manuscript.

\section{REFERENCES}

1. Bastos HN, Osorio NS, Gagneux S, Comas I, Saraiva M. The Troika HostPathogen-Extrinsic Factors in Tuberculosis: Modulating Inflammation and Clinical Outcomes. Front Immunol (2017) 8:1948. doi: 10.3389/ fimmu.2017.01948

2. Zumla A, Rao M, Parida SK, Keshavjee S, Cassell G, Wallis R, et al. Inflammation and Tuberculosis: Host-Directed Therapies. I Intern Med (2015) 277(4):373-87. doi: 10.1111/joim.12256

3. Dorhoi A, Kaufmann SH. Perspectives on Host Adaptation in Response to Mycobacterium Tuberculosis: Modulation of Inflammation. Semin Immunol (2014) 26(6):533-42. doi: 10.1016/j.smim.2014.10.002

4. Sproston NR, Ashworth JJ. Role of C-Reactive Protein at Sites of Inflammation and Infection. Front Immunol (2018) 9:754. doi: 10.3389/ fimmu.2018.00754

5. Andersen CBF, Stodkilde K, Saederup KL, Kuhlee A, Raunser S, Graversen JH, et al. Haptoglobin. Antioxid Redox Signal (2017) 26(14):814-31. doi: 10.1089/ars.2016.6793

6. Cater JH, Wilson MR, Wyatt AR. Alpha-2-Macroglobulin, a HypochloriteRegulated Chaperone and Immune System Modulator. Oxid Med Cell Longev (2019) 2019:5410657. doi: 10.1155/2019/5410657

7. Bottazzi B, Inforzato A, Messa M, Barbagallo M, Magrini E, Garlanda C, et al. The Pentraxins PTX3 and SAP in Innate Immunity, Regulation of Inflammation and Tissue Remodelling. J Hepatol (2016) 64(6):1416-27. doi: 10.1016/j.jhep.2016.02.029

8. Lawn SD, Kerkhoff AD, Vogt M, Wood R. Diagnostic and Prognostic Value of Serum C-Reactive Protein for Screening for HIV-Associated Tuberculosis. Int J Tuberc Lung Dis (2013) 17(5):636-43. doi: 10.5588/ijtld.12.0811

9. Drain PK, Mayeza L, Bartman P, Hurtado R, Moodley P, Varghese S, et al. Diagnostic Accuracy and Clinical Role of Rapid C-Reactive Protein Testing in HIV-Infected Individuals With Presumed Tuberculosis in South Africa. Int J Tuberc Lung Dis (2014) 18(1):20-6. doi: 10.5588/ijtld.13.0519

10. Wilson D, Badri M, Maartens G. Performance of Serum C-Reactive Protein as a Screening Test for Smear-Negative Tuberculosis in an Ambulatory High HIV Prevalence Population. PloS One (2011) 6(1):e15248. doi: 10.1371/ journal.pone.0015248

11. Yoon C, Semitala FC, Atuhumuza E, Katende J, Mwebe S, Asege L, et al. PointOf-Care C-Reactive Protein-Based Tuberculosis Screening for People Living

\section{FUNDING}

This project has been funded in whole or in part with Federal funds from the Government of India's (GOI) Department of Biotechnology (DBT), the Indian Council of Medical Research (ICMR), the United States National Institutes of Health (NIH), National Institute of Allergy and Infectious Diseases (NIAID), Office of AIDS Research (OAR), and distributed in part by CRDF Global [grant USB1-31149-XX-13]. This work is also funded by CRDF Global RePORT India Consortium Supplemental Funding [grant OISE-17-62911-1].

\section{ACKNOWLEDGMENTS}

We thank the staff of Department of Clinical Research and the Department of Bacteriology, NIRT for valuable assistance in bacterial cultures and radiology and the staff of MVDRC, RNTCP and Chennai corporation for valuable assistance in recruiting the patients for this study. Data in this manuscript were collected as part of the Regional Prospective Observational Research for Tuberculosis (RePORT) India Consortium.

With HIV: A Diagnostic Accuracy Study. Lancet Infect Dis (2017) 17 (12):1285-92. doi: 10.1016/S1473-3099(17)30488-7

12. Bedell RA, van Lettow M, Meaney C, Corbett EL, Chan AK, Heyderman RS, et al. Predictive Value of C-Reactive Protein for Tuberculosis, Bloodstream Infection or Death Among HIV-Infected Individuals With Chronic, nonSpecific Symptoms and Negative Sputum Smear Microscopy. Trop Med Int Health (2018) 23(3):254-62. doi: 10.1111/tmi.13025

13. Shapiro AE, Hong T, Govere S, Thulare H, Moosa MY, Dorasamy A, et al. CReactive Protein as a Screening Test for HIV-Associated Pulmonary Tuberculosis Prior to Antiretroviral Therapy in South Africa. AIDS (2018) 32(13):1811-20. doi: 10.1097/QAD.0000000000001902

14. Bapat PR, Satav AR, Husain AA, Shekhawat SD, Kawle AP, Chu JJ, et al. Differential Levels of Alpha-2-Macroglobulin, Haptoglobin and SeroTransferrin as Adjunct Markers for TB Diagnosis and Disease Progression in the Malnourished Tribal Population of Melghat, India. PloS One (2015) 10 (8):e0133928. doi: 10.1371/journal.pone.0133928

15. Pai M, Behr MA, Dowdy D, Dheda K, Divangahi M, Boehme CC, et al. Tuberculosis. Nat Rev Dis Primers (2016) 2:16076. doi: 10.1038/nrdp.2016.76

16. Kumar NP, Moideen K, Nancy A, Viswanathan V, Thiruvengadam K, Nair D, et al. Plasma Chemokines are Baseline Predictors of Unfavorable Treatment Outcomes in Pulmonary Tuberculosis. Clin Infect Dis (2020) 7:ciaa1104. doi: $10.1093 / \mathrm{cid} /$ ciaal 104

17. Kumar NP, Moideen K, Nancy A, Viswanathan V, Thiruvengadam K, Sivakumar $\mathrm{S}$, et al. Association of Plasma Matrix Metalloproteinase and Tissue Inhibitors of Matrix Metalloproteinase Levels With Adverse Treatment Outcomes Among Patients With Pulmonary Tuberculosis. JAMA Netw Open (2020) 3(12): e2027754. doi: 10.1001/jamanetworkopen.2020.27754

18. Goletti D, Lindestam Arlehamn CS, Scriba TJ, Anthony R, Cirillo DM, Alonzi T, et al. Can We Predict Tuberculosis Cure? What Tools are Available? Eur Respir J (2018) 52(5):1801089. doi: 10.1183/13993003.01089-2018

19. Kornfeld H, West K, Kane K, Kumpatla S, Zacharias RR, Martinez-Balzano C, et al. High Prevalence and Heterogeneity of Diabetes in Patients With TB in South India: A Report From the Effects of Diabetes on Tuberculosis Severity (EDOTS) Study. Chest (2016) 149(6):1501-8. doi: 10.1016/ j.chest.2016.02.675

20. Rockwood N, du Bruyn E, Morris T, Wilkinson RJ. Assessment of Treatment Response in Tuberculosis. Expert Rev Respir Med (2016) 10(6):643-54. doi: $10.1586 / 17476348.2016 .1166960$ 
21. Wallis RS, Kim P, Cole S, Hanna D, Andrade BB, Maeurer M, et al. Tuberculosis Biomarkers Discovery: Developments, Needs, and Challenges. Lancet Infect Dis (2013) 13(4):362-72. doi: 10.1016/S1473-3099(13)70034-3

22. Yoon C, Chaisson LH, Patel SM, Allen IE, Drain PK, Wilson D, et al. Diagnostic Accuracy of C-Reactive Protein for Active Pulmonary Tuberculosis: A Meta-Analysis. Int J Tuberc Lung Dis (2017) 21(9):1013-9. doi: 10.5588/ijtld.17.0078

23. Pavan Kumar N, Anuradha R, Andrade BB, Suresh N, Ganesh R, Shankar J, et al. Circulating Biomarkers of Pulmonary and Extrapulmonary Tuberculosis in Children. Clin Vaccine Immunol (2013) 20(5):704-11. doi: 10.1128/ CVI.00038-13

24. Wong CT, Saha N. Changes in Serum Proteins (Albumin, Immunoglobulins and Acute Phase Proteins) in Pulmonary Tuberculosis During Therapy. Tubercle (1990) 71(3):193-7. doi: 10.1016/0041-3879(90)90075-J

25. Jacobs R, Malherbe S, Loxton AG, Stanley K, van der Spuy G, Walzl G, et al. Identification of Novel Host Biomarkers in Plasma as Candidates for the Immunodiagnosis of Tuberculosis Disease and Monitoring of Tuberculosis Treatment Response. Oncotarget (2016) 7(36):57581-92. doi: 10.18632/ oncotarget.11420

26. Phalane KG, Kriel M, Loxton AG, Menezes A, Stanley K, van der Spuy GD, et al. Differential Expression of Host Biomarkers in Saliva and Serum Samples From Individuals With Suspected Pulmonary Tuberculosis. Mediators Inflamm (2013) 2013:981984. doi: 10.1155/2013/981984

27. Furman D, Campisi J, Verdin E, Carrera-Bastos P, Targ S, Franceschi C, et al. Chronic Inflammation in the Etiology of Disease Across the Life Span. Nat Med (2019) 25(12):1822-32. doi: 10.1038/s41591-019-0675-0
28. Racanelli AC, Kikkers SA, Choi AMK, Cloonan SM. Autophagy and Inflammation in Chronic Respiratory Disease. Autophagy (2018) 14(2):22132. doi: $10.1080 / 15548627.2017 .1389823$

Author Disclaimer: The contents of this publication are solely the responsibility of the authors and do not represent the official views of the DBT, the ICMR, the $\mathrm{NIH}$, or CRDF Global. This work was also funded in part by the Division of Intramural Research, NIAID, NIH.

Conflict of Interest: The authors declare that the research was conducted in the absence of any commercial or financial relationships that could be construed as a potential conflict of interest.

Publisher's Note: All claims expressed in this article are solely those of the authors and do not necessarily represent those of their affiliated organizations, or those of the publisher, the editors and the reviewers. Any product that may be evaluated in this article, or claim that may be made by its manufacturer, is not guaranteed or endorsed by the publisher.

Copyright ( $(2021$ Kumar, Moideen, Nancy, Viswanathan, Thiruvengadam, Sivakumar, Hissar, Kornfeld and Babu. This is an open-access article distributed under the terms of the Creative Commons Attribution License (CC BY). The use, distribution or reproduction in other forums is permitted, provided the original author(s) and the copyright owner(s) are credited and that the original publication in this journal is cited, in accordance with accepted academic practice. No use, distribution or reproduction is permitted which does not comply with these terms. 